\section{Verordnungen und Vorschriften in der medizinischen Laboratoriumsdiagnostik}

\author{
W. G. Guder \\ München, Deutschland
}

Englischer Begriff order/decree; regulations; directives

Definition Durch Gesetze oder übergeordnete Instanzen geregelte Direktiven zur Durchführung eines Vorgangs (hier in der Laboratoriumsmedizin).

Beschreibung Die in der präanalytischen Phase ( $\triangleright$ Präanalytische Phase) der $>$ Laboratoriumsmedizin geltenden gesetzlichen Regelungen und Vorschriften betreffen im Wesentlichen den Umgang mit potenziell infektiösem Material beim Bearbeiten, Versand und der Weitergabe $(\triangleright$ Versand von Proben) sowie die Gefahrenstoffverordnungen zur Vermeidung von Schädigungen der damit betrauten Personen $(\triangleright \mathrm{Ge}-$ fahrstoffpiktogramme).

Die in der Präanalytik verwendeten Materialien unterliegen Vorschriften der Standardisierung und Normen, die durch EN-Normen beschrieben sind. Im Bereich des Qualitätsmana- gements ( $\triangleright$ Qualitätsmanagement) sind ebenso wie bei der Durchführung der externen Qualitätssicherung ( $\vee$ Qualitätssicherung, externe) EN-Normen zur Durchführung auch präanalytischer Prozesse enthalten.

Bei Bluttransfusionen sind die Richtlinien zur Immunhämatologie und das Transfusionsgesetz, bei allen anderen medizinischen Untersuchungen die $>$ Richtlinie der Bundesärztekammer zur Qualitätssicherung laboratoriumsmedizinischer Untersuchungen relevant.

\section{Literatur}

Bundesärztekammer (1992) Richtlinien der Bundesärztekammer zur Qualitätssicherung in der Immunhämatologie, in der Mikrobiologie. http://www.bundesaerztekammer.de/Richtlinien/Labor (Jan 2018)

Bundesärztekammer (2008) Richtlinie der Bundesärztekammer zur Qualitätssicherung laboratoriumsmedizinischer Untersuchungen. Dt Ärztebl 105:C301-C315

EN 829 (1996) ) In vitro diagnostic systems. Transport packages for medical and biological specimens. Requirements, tests. European Committee for Standardization (CEN), Brüssel

DIN, EN, ISO 15189 (2007) Medizinische Laboratorien - Besondere Anforderungen an die Qualität und Kompetenz. Beuth-Verlag, Berlin

Gesetz zur Regelung des Transfusionswesens (2012). http://www. gesetze-im-internet.de/tfg/. Zugegriffen im Jan 2018 